\title{
Segmentation of gated Tl-SPECT images and computation of ejection fraction: A different approach
}

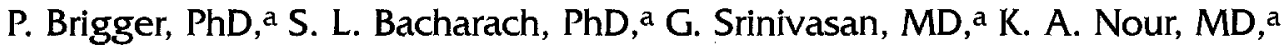 \\ J. A. Carson, BS, ${ }^{a}$ V. Dilsizian, MD, ${ }^{a}$ A. Aldroubi, PhD, ${ }^{b}$ and $M$. Unser, $\mathrm{PhD}^{\mathrm{c}}$
}

Background. We describe a set of image processing algorithms and mathematical models that can be advantageously used in schemes for the segmentation of thallium-201-single photon emission computed tomography (SPECT) images and for computation of left ventricular ejection fraction (EF).

Methods. The system consists of two independent blocs for image segmentation and computation of function. The former is based on a multiresolution elliptical coordinate transformation and dynamic contour tracking. Computation of EF is formulated on the basis of both the endocardial and epicardial contours, and we compare this formulation with that using only the endocardial border for images with low signal-to-noise ratios. The accuracy of border detection was validated against manual border tracing on FDG-PET images, simulated Tl-201-SPECT images where the true underlying borders were known, and actual Tl-201-SPECT images. Finally, we compared EFs computed for FDG-PET, technetium-99m-SPECT and Tl-201-SPECT with those obtained from planar gated blood pool imaging.

Results. The automatically obtained results always were within the manual uncertainty range. Agreement between myocardial volumes from positron emission tomography and automatically obtained values from the simulated Tl-201-SPECT images was excellent $(r=0.95$, $n=32$ ). Agreement between EFs from planar gated blood pool imaging and the other image modalities was good (FDG-PET: $y=5.89+1.21 \mathrm{x}, r=0.92$, see $=6.24, n=19$, Tc-99m-SPECT: $y=-3.86+1.06 x, r=0.88$, see $=7.78, n=9$, Tl-201-SPECT: $y=17.8+0.81 \mathrm{x}, r=0.77$, see $=7.44$, $n=26$ ). For noisy input data the combined use of information from epicardial and endocardial contours gives more accurate EF values than the traditional formula on the basis of the endocardial contour only.

Conclusions. Alternate approaches for segmentation and computation of function have been presented and validated. They might also be advantageously incorporated into other existing techniques. (J Nucl Cardiol 1999;6:286-97.)

Key Words: Tl-201 SPECT • gated blood pool imaging • FDG PET

Gated perfusion single photon emission computed tomography (SPECT) studies are important because they permit simultaneous measurement of both function and perfusion. A considerable body of research has shown that technetium-99m-mibi SPECT can be used for automatic computation of ejection fraction (EF). ${ }^{1-5}$ The high noise present in gated thallium-201-SPECT studies has made global function measurements more problematic, and although some data are available supporting its use

From the aNational Institutes of Health, Bethesda, Md, bVanderbilt University, Nashville, Tenn, and the ${ }^{\mathrm{c} S}$ wiss Federal Institute of Technology, Lausanne, Switzerland.

Supported in part by the Swiss National Research Foundation.

Received for publication Feb 3, 1998; revision accepted Aug 13, 1998. Reprint requests: Stephen L. Bacharach, PhD, BIdg 10, Rm 1C401,

National Institutes of Health, Bethesda, MD 20892-1180; Steve_Bacharach@nih.gov.

Copyright (1) 1999 by the American Society of Nuclear Cardiology. 1071-3581/99/\$8.00+0 $\quad \mathbf{4 3 / 1 / 9 4 1 1 7}$ for functional measurements, ${ }^{6}$ gating has not been widely adopted for T1-201-SPECT. In this study we focus on these more noisy Tl-201-SPECT images. We introduce an image processing methodology and new mathematical models for segmentation and computation of function that might be advantageously incorporated into other (already existing) algorithms as well. We investigate a method that has two unique features. First, the technique uses a set of robust and general image processing algorithms that rely on a minimal number of user-defined input parameters, and therefore no parameter adjustments are necessary for input data over a large range of noise levels. We have tested the method by comparing its results with those obtained from manually drawn contours and with the results obtained from gated blood pool (GPB) imaging. Second, we investigate a method for computation of EF on the basis of a combined use of both endocardial and epicardial border information. It is 

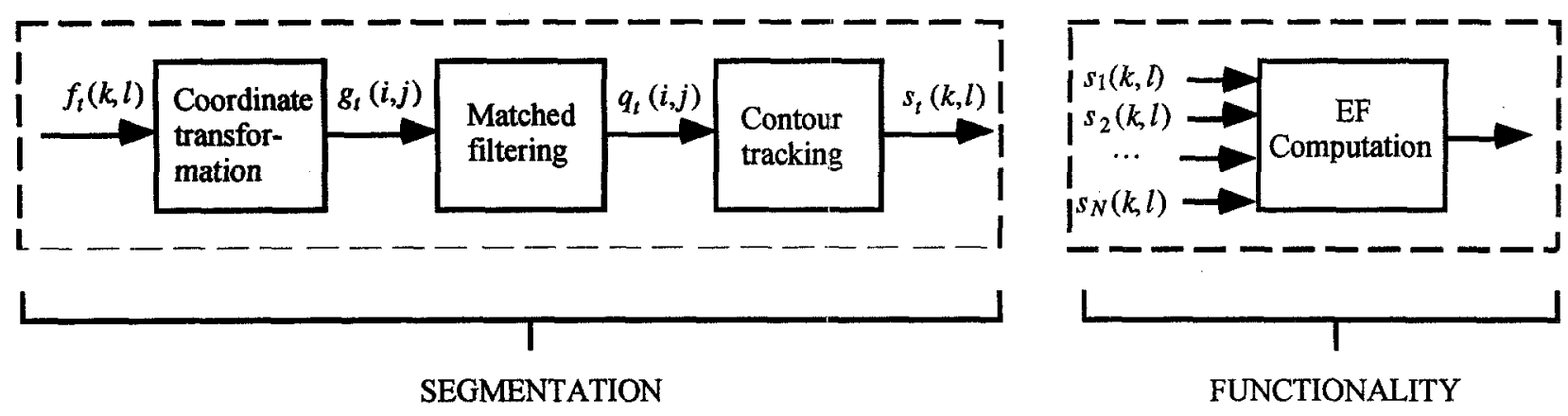

Figure 1. Block diagram of image processing system.

hypothesized that by including the epicardial border information, a more reliable estimate of $\mathrm{EF}$ might be obtained, because much of the epicardial surface has better contrast than the corresponding endocardial surface, because of the absence of left ventricular (LV) cavity activity. Moreover, there are fewer confounding structures (eg, papillary muscles) that influence the epicardial border. We test this hypothesis by comparing results with and without the additional epicardial information.

\section{METHODS}

\section{Image Processing System}

The purpose of this section is to present the different components of the image processing system depicted in Figure 1. The following nomenclature has been chosen to describe the images at the various stages of processing: $f_{t}(k, l)$ : original, discrete image; $g_{t}(i, j)$ : area of interest in the transformed coordinate system; $q_{t}(i, j)$ : image of enhanced endocardial or epicardial boundaries; $s_{t}(k, l)$ : segmented image.

The subscript $t$ denotes the gating interval. The variables $k$ and $l$ denote the pixel coordinates in the Cartesian coordinate system, whereas the variables $i$ and $j$ describe the location in a transformed coordinate system. In what follows, we will first describe the principal elements of the segmentation bloc, explain how they are used for a successful segmentation, and then describe the functionality bloc.

\section{Elliptical Coordinate Transformation}

The main purpose of a change of coordinate system is to present the data in a form suitable for the contour tracking algorithm described below and to provide a system in which true count profiles across the myocardium can be measured. These profiles should be perpendicular to the heart wall.

In our study, we have used long-axis views because they contain maximal information in the apical region. In this view the shape of the myocardium suggests the use of an elliptical coordinate system. If the myocardium were indeed elliptical, this coordinate transformation would project it into an image
A
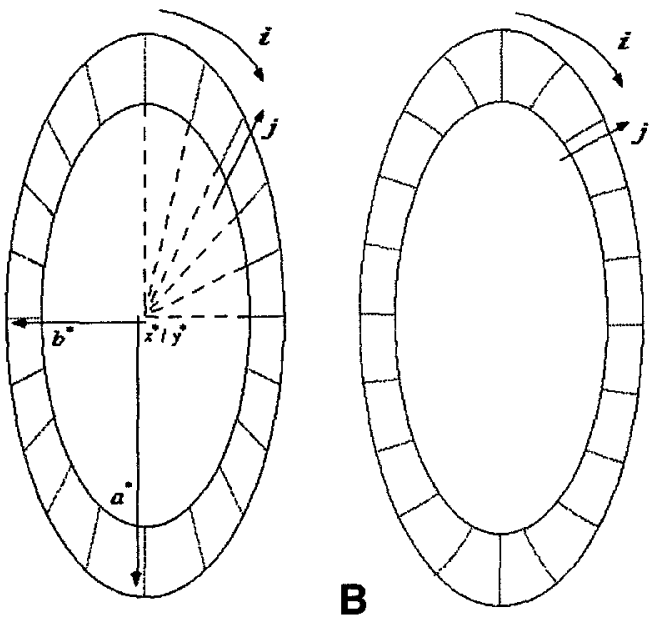

Figure 2. Comparison between (A) polar and (B) elliptic coordinate system.

where the endocardial and epicardial boundaries were straight lines. Note that the accuracy of the proposed algorithm does not depend on the myocardium actually being elliptical-it is simply a mathematical convenience. The horizontal representation will facilitate the subsequent steps of filter matching and contour tracking in the processing system because the algorithms can rely on operators that act along lines and columns instead of radial parameters. The geometrical transformation maps the original image $f_{t}(k, l), k, l \varepsilon Z$ into an area of interest $g_{t}(i, j), i, j \varepsilon Z$ in the transformed coordinate system ( $Z$ is the set of all integers). The parameter $i$ relates to the radial angle, and $j$ is an index proportional to the distance from the ellipse center along an elliptical path $r(p, q)$. This path is computed such that it is perpendicular at all times to the ellipse. This is in contrast to the traditional radial path that computes count profiles across the myocardium at oblique angles (Figure 2). Such a feature is potentially useful in all applications measuring perfusion profiles. ${ }^{7-14}$

The ellipse is characterized by 5 parameters: center coordinates $\left(x_{0}, y_{0}\right)$, long- and short-axis $\left(a_{0}, b_{0}\right)$, and orientation $\left(\theta_{0}\right)$. Because of its ability to detect complex patterns, we have chosen the Hough transform ${ }^{14}$ for the detection of the ellipse. Every variable in the analytical curve model defines 1 dimension in the multidimensional Hough space. The Hough space is then scanned for all possible parameter values. Hence, each bin 

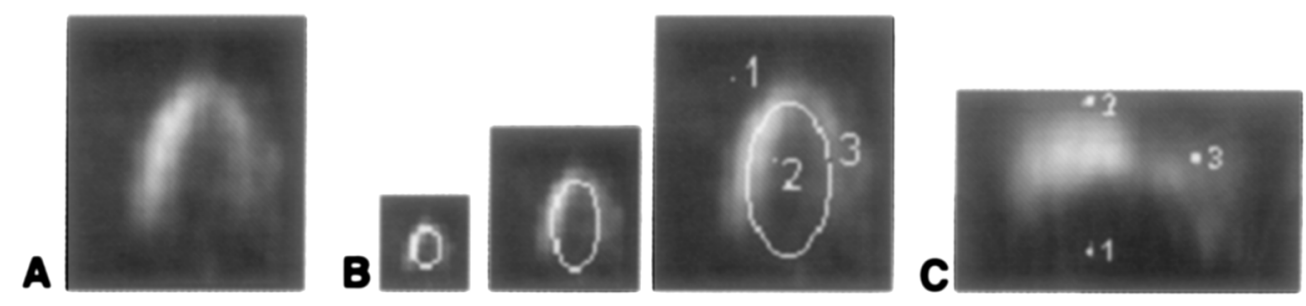

Figure 3. (A) Original non-gated image, (B) multi-resolution Hough algorithm: size of original image is divided by 2 at each level. Ellipse is detected in smallest image, the result up-projected onto next larger image, refined, up-projected again onto original image, and refined, (C) transformed image. Numbered points ( $I$ to 3$)$ illustrate mapping of three distinct points.

in the 5-dimensional Hough space represents the parameters of exactly 1 ellipse. One increments each bin with the gray-level value of the image pixels covered by the model. The bin with the maximal count represents the parameters of the unknown curve model. The main computational difficulty is the huge size of the Hough parameter space. To circumvent this problem, we have implemented the algorithm within a multiresolution environment. ${ }^{15}$ With this implementation, the Hough space is scanned in a spatially reduced image. The parameters of the best fit ellipse at the coarse resolution level are detected and are then symmetrically up-projected onto the next finer image level, where the optimal result is situated in the neighborhood of the up-projected pixels. Hence, it is not necessary anymore to search through the entire image, but only through a small portion of it. We have optimized the pyramid decomposition by use of centered cubic splines, which provide a faithful representation of the original image at coarse pyramid levels. Without a multiresolution approach, computation time is 2.8 hours for a $128 \times 128$ image on a PowerPC, model 7600, 120 MHz. This time compares to 53 seconds, when two coarser resolution levels are used (ie, number of columns and lines divided by $4\left[2^{2}\right]$ ). In addition, the multiresolution approach favorably smoothes the images and reduces noise. The results of the various processing steps are illustrated in Figure 3.

\section{Matched Filter}

Because of finite resolution acquisition devices and partial volume effects, ${ }^{16}$ the perfusion profiles are bell-shaped rather than rectangular. The mid-myocardial contour must be assumed to lie close to the maximum of the bell shape, the epicardial contour lies between the transition from dark to bright, and the endocardial boundary lies between the transition from bright to dark, assuming one moves in the direction of decreasing radii.

We will treat the detection of each of the contours as a separate problem. The matched filtering problem is to find a linear filter that enhances the transition to be detected. ${ }^{17}$ Because the change of coordinate system is intended to flatten the myocardium horizontally, the filtering is performed selectively in the vertical dimension in the transformed image.

We chose to use the simplest possible enhancement filters: convolution operators of the form

Endocardial contour: $q_{t}(i, j)=g_{t}(i, j-1)-g_{t}(i, j+1)$

Epicardial contour: $q_{t}(i, j)=g_{t}(i, j+1)-g_{t}(i, j-1)$

with equation $1=-$ equation 2 . These simple gradient operators enhance transitions from high to low pixel for the endocardial boundary and transitions from low to high pixel values for the epicardial boundary. Other enhancement schemes could have been used, such as through an experimental determination of the transition characteristics for a particular boundary. 18 However, such procedures would yield different convolution operators for image acquisitions of different types (ie, T1201-SPECT, Tc-99m-SPECT, or FDG-PET) or for different pre-reconstruction filters. The contour tracking algorithm described below is by design very robust, and the exact shape of the convolution operator has little effect on the segmentation result. Therefore, by use of the simple enhancement operators in equations 1 and 2 above, a single tracking algorithm can be used on a large class of images, as will be demonstrated in the Results section.

\section{Contour Tracking}

Segmentation and wall thickening measurements of perfusion SPECT images have almost exclusively been based on one of the following three techniques: (1) Counts-based segmentation, used in positron emission tomography (PET) ${ }^{12}$ and SPECT, ${ }^{19-21}$ relating maximal counts in a radial count profile to the wall thickness. The technique is linear only for small wall thickness with respect to the point spread function of the acquisition device. ${ }^{8}$ (2) Geometry-based segmentation, ${ }^{7}$ performing a local threshold at half maximum of the count profile. This technique operates in the linear domain for wall dimensions larger than the resolution of the acquisition device. Unfortunately, both techniques are highly nonlinear precisely in the heart's dimensions. ${ }^{16}$ Variations and combinations of both techniques 22 or model-based techniques ${ }^{10}$ may be used to obtain more stable and consistent segmentation results. (3) Global thresholding, which gives only incomplete and very inaccurate boundaries because of nonhomogeneous count profiles. All three techniques require images with relatively good uptake, which is often not the case in practice.

For our application, we must design an algorithm that is robust in noisy environments and detects a single, smooth, and connected contour (endocardial and epicardial boundaries are considered as two separate problems). The contour detection is carried out in the transformed coordinate system and consists basically in finding a path that goes from the left side of the image to the right side, maximizing some criterion. We use the simplest possible figure of merit, that is, the cumulative sum of gray level values along the contour segment, denoted by $\zeta\left(\mathrm{T}_{\mathrm{k}}\right)$. 


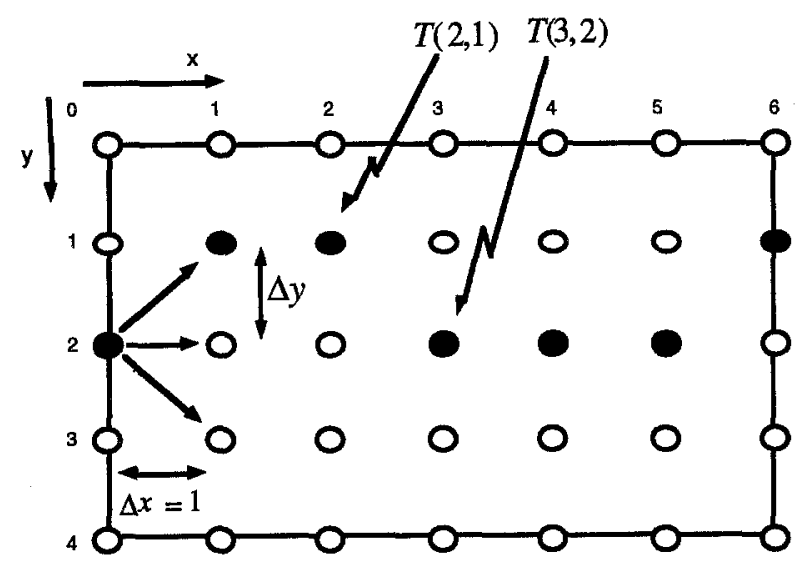

Figure 4. Black points indicate contour segment $T=\left[\left(1, j_{1}\right),(2\right.$, $\left.\left.\mathrm{j}_{2}\right), \ldots,\left(\mathrm{i}, \mathrm{j}_{\mathrm{i}}\right), \ldots,\left(\mathrm{N}, \mathrm{j}_{\mathrm{N}}\right)\right]$ in transformed coordinate system.

The problem can be reformulated as an optimization problem. We search for a contour segment $T=\left[\left(1, j_{l}\right),\left(2, j_{2}\right), \ldots,(i\right.$, $\left.\left.j_{i}\right), \ldots,\left(N, j_{N}\right)\right]$, satisfying the constraints and maximizing $\zeta\left(T_{k}\right)$. An example of a contour $T$ is given in Figure 4 . The horizontal coordinate in the transformed coordinate system of the contour increases by one as we move from one point on $\mathrm{T}$ to the next. The vertical coordinate displacement is restricted to some maximal value $\Delta y$ pixels. Typically, $1 \leq \Delta y \leq 3$ pixels are adequate. This restriction defines our smoothness constraint. This same procedure-maximizing the sum of gray level values along the contour (ie, maximizing $\zeta\left[\mathrm{T}_{\mathrm{k}}\right]$ ) -is also applied to "gaps" formed by perfusion or metabolism defects. This procedure, combined with the smoothness constraint above, is designed to make the contour track smoothly across even severe defects (even severe defects usually contain $>30 \%$ of normal uptake). In the limiting case of zero activity in the gap (a situation that never occurs in practice), the smoothness constraint would still force the algorithm to smoothly interpolate across the defect to the neighboring non-zero portions of the contour.

Given these definitions, the optimal path can in principle be determined by enumerating all allowable contour segments, evaluating their respective figure or merits and choosing the one that maximizes $\zeta\left(\mathrm{T}_{\mathrm{k}}\right)$. Such a procedure would quickly result in unfeasible computational requirements. A better solution is found through dynamic programming. ${ }^{23}$ The technique exploits the fact that if the best path goes through a given point $(i, j)$, then this path includes as a portion of it, the best path to the point $(i, j)$. Hence, for each point $(i, j)$, one stores the predecessor point such that $\zeta\left(T_{k}\right)$ is maximized and records the updated value of $\zeta\left(T_{k}\right)$. At the end of the cycle, the optimal figure of merit is found by searching for the maximum of $\zeta_{i=N, j}^{*}$ and retrieving the optimal trajectory by backtracking. Such an algorithm is extremely efficient and requires a fraction of a second of computation time for an image of size $128 \times 128$. Although the algorithm produces globally smooth contours, it may still present local spikes of maximal deviation of $\Delta y$. We eliminate those by a post-processing smoothing of the contours in the transformed coordinate system by use of a Gaussian filter. An example for the tracking of the epicardial contour is given in Figure 5.

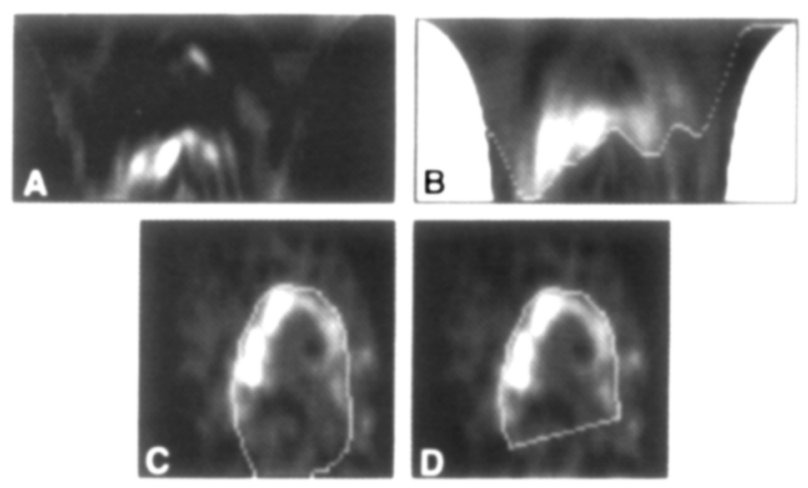

Figure 5. (A) Enhanced epicardial image in transformed coordinates system (negative values set to black), (B) tracked contour, (C) epicardial contour in original image, (D) epicardial contour with base line.

\section{Putting it All Together}

On the basis of the three image processing elements, computation of the endocardial and epicardial boundaries is as follows. For each slice the nongated image is computed by summing all gating intervals. On this image the elliptical parameters (center, axis length, orientation) are computed, which are assumed to be identical for all frames within 1 slice. For contour tracking, we chose a smoothing value $\Delta y=2$ pixels. If $\Delta y$ were zero, we would find a straight line corresponding to a perfect ellipse. Contour tracking is carried out in the images obtained from the respective matched filters to enhance endocardial and epicardial contours. The dynamic contour tracking algorithm with its built-in smoothing properties ensures excellent performance over perfusion defects by interpolation between the surrounding more normal segments (see http://picasso.ncrr.nih.gov/brigger/Spect for practical examples)

Finally, to prevent erroneous segmentation in frames with very low perfusion uptake, we first compute the endocardial and epicardial boundary in the summed, nongated image. We expand the epicardial contour by one pixel, and we shrink the endocardial contour by one pixel. Those contours then serve as outer and inner limits for all contours in the slice.

For each slice and at each time point, the valve plane has been determined manually. In this study, we were mainly interested in studying the ability of the proposed concept to accurately determine contours of the left ventricle and to demonstrate reproducibility and reliability of the proposed techniques. Although valve plane selection is important, ${ }^{24}$ automatic valve plane selection methods are already available and have been described elsewhere. ${ }^{4,5}$

\section{Functionality Bloc}

The functionality bloc receives as input the absolute values of the volume of the endocardium and epicardium for each time point $t$. Ejection fraction can then be computed as

$$
\mathrm{EF}=\frac{V_{\text {endo }}\left(t_{D}\right)-V_{\text {endo }}\left(t_{S}\right)}{V_{\text {endo }}\left(t_{D}\right)}
$$




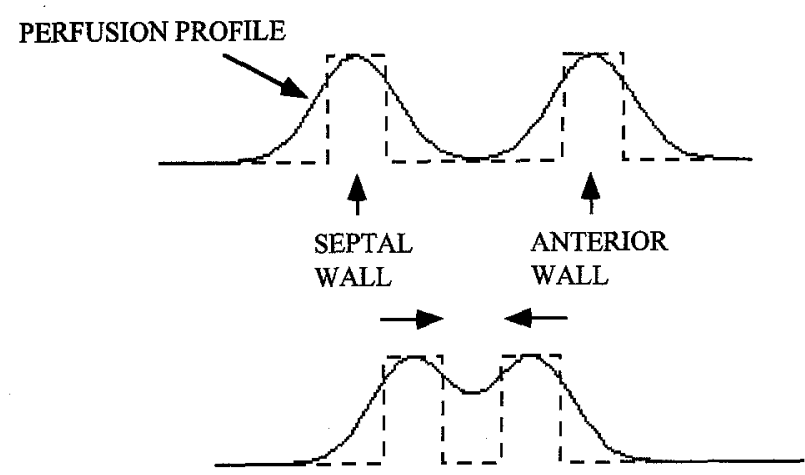

Figure 6. Illustration of interference of perfusion profiles of opposite myocardial walls during systole.

where $\mathrm{V}_{\text {endo }}\left(\mathrm{t}_{\mathrm{D}}\right)=\max _{t}\left[V_{\text {endo }}(t)\right]$ and $V_{\text {endo }}\left(t_{S}\right)=\min _{t}\left[V_{\text {endo }}(t)\right]$ are the end-diastolic and -systolic volumes.

Equation 3 is not optimal for two reasons: (1) It is extremely sensitive to noise because the result is based on only two values out of the $\mathrm{N}_{t}$ gating intervals (the maximum and minimum value, respectively). (2) The endocardial boundary is difficult to identify accurately. This is because at low resolution, perfusion profiles of opposite walls may spill over when the walls are close (eg, at systole) and also because of the possible presence of papillary muscle and bloodpool activity in the LV cavity. A schematic illustration is given in Figure 6.

We can reduce the effect of noise in the data (such as found in data from Tl-201-SPECT images) by considering all gating intervals. It has been reported elsewhere ${ }^{25}$ that for computation of $\mathrm{EF}$, use of 2 Fourier harmonics to fit the volume curve gave $<3 \%$ bias and minimum total error compared with other numbers of harmonics over a wide range of noise levels in the volume curve. Hence, systole and diastole were taken here as the minimum and maximum of the 2 Fourier harmonic fitted data.

Addressing the second point above, we propose a method that uses both the endocardial and epicardial contour to compute EF. The epicardial border is easier to identify than the endocardial one-outside influence from structures such as right ventricle or spleen may still alter septal perfusion, but in general, in the long axis, the epicardial free wall and apical wall have better contrast, and there are fewer confounding structures such as papillary muscles. The endocardial volume can be rewritten as $V_{\text {endo }}(t)=V_{e p i}(t)-m$, where $m$ is the volume of the myocardium, and therefore $\mathrm{EF}$ is

$$
\mathrm{EF}=\frac{V_{e p i}\left(t_{D}\right)-V_{e p i}\left(t_{S}\right)}{V_{e p i}\left(t_{D}\right)-m}
$$

Myocardial mass is conserved over the cycle. Assuming tissue density is also nearly constant, myocardial volume $m$ is also conserved and was obtained by averaging the difference between epicardial and endocardial volume over the entire cycle. The percent standard deviation from the mean was $10 \%$ in average $(n=30)$. Therefore the effect of outliers in the endocardial volume is reduced, and the precision of measuring $m$ is increased by as much as a factor of $\sqrt{N_{t}-2}$. It requires experimental verification (performed below) to determine the net effect of using this epicardial-based method (equation 4) rather than the usual endocardial method (equation 3) on the variance in EF. The reduction in variance in EF caused by use of the (hypothesized easier to measure) epicardial rather than the endocardial surface is partially offset by an additional variance term resulting from variance in $m$. It was hypothesized that because this variance in $m$ could be reduced by averaging over the cardiac cycle, as described above, that the net error in EF would be reduced.

\section{Image Acquisition, Image Processing, and Patient Population}

To test the algorithm and evaluate its performance, 3 different image modalities with different image characteristics were considered. (1) Gated FDG-PET images were obtained for 19 subjects ( 16 men, 3 women) with $5 \mathrm{mCi}$ of $18 \mathrm{~F}$ fluorodeoxyglucose (FDG), with a GE-Advance PET scanner used in 3-dimensional (ie, septa out) mode with scatter and attenuation correction. Images were acquired for 30 minutes 30 minutes after injection. Slice separation was $4.25 \mathrm{~mm}$, inplane resolution $\sim 7 \mathrm{~mm}$, and pixel size was $2 \mathrm{~mm}$. All subjects treated with FDG had known or suspected coronary artery disease. Only 2 of the 19 subjects had no FDG defects. Seventeen of 19 subjects had a total of 23 defects, with 13 of these 23 being categorized as nonviable tissue ( $<50 \%$ FDG uptake). (2) Gated Tc$99 \mathrm{~m}$-sestamibi SPECT images were obtained for 9 individuals (6 men, 3 women) with known or suspected coronary artery disease, with a triple-detector Trionix Co "Triad" camera $(\sim 10$ $\mathrm{mCi}$, studied at rest). In-plane reconstructed resolution was $\sim 12$ $\mathrm{mm}$ with $3.6 \mathrm{~mm}$ pixel size (Butterworth filter, cutoff 0.76 , rolloff 3.0). Four of the 9 had abnormal rest perfusion studies with 6 defects in all. (3) Gated Tl-201 SPECT images were obtained for 26 subjects on a 90-degree, 2-headed ADAC Vertex with $3.2 \mathrm{~mm}$ pixels and an image resolution of $\sim 14.5 \mathrm{~mm}$, (Butterworth filter, cutoff 0.50 , rolloff 3.0 , with $\sim 2$ to $3 \mathrm{mCi}$ injected during peak stress, and imaged shortly thereafter). All 26 subjects treated with T1 SPECT (20 men, 6 women) had known or suspected coronary artery disease and were being evaluated with the following protocol: $3 \mathrm{mCi}$ Tl-201 injection at peak exercise - redistribution $(\sim 3$ to 4 hours after injection) - $1 \mathrm{mCi}$ reinjection (15 minutes later still). The 26 gated $\mathrm{Tl}$ image sets used in this study were all from the reinjection studies. Eight of the 26 subjects had no defects at reinjection, whereas 18 of 26 had significant defects. Among the 18 subjects with significant defects, there were 22 defects in all, of which 9 (in 8 different subjects) were categorized visually as severe irreversible defects.

Note that both Tl-201 and Tc-99m-MIBI used higher resolution reconstruction filters than was used in many laboratories. Acquisition consisted of 8 or 10 gating intervals over each RR cycle for Tl-201 and Tc-99m-MIBI, and 16 for PET. Four long-axis slices were created by slicing the short-axis set at $-22.5^{\circ}, 22.5^{\circ}, 67.5^{\circ}$, and $112.5^{\circ}$ degrees, where 0 degrees $=12$ $0^{\prime}$ 'clock. The first two of these could be considered vertical long-axis slices, the second two horizontal long-axis slices. This resulted in a total of 32 or 40 images ( 64 for PET). Rotation of each slice about its vertical axis in the original image and averaging of the four slices allows computation of cavity volumes from the contours. The patient population con- 

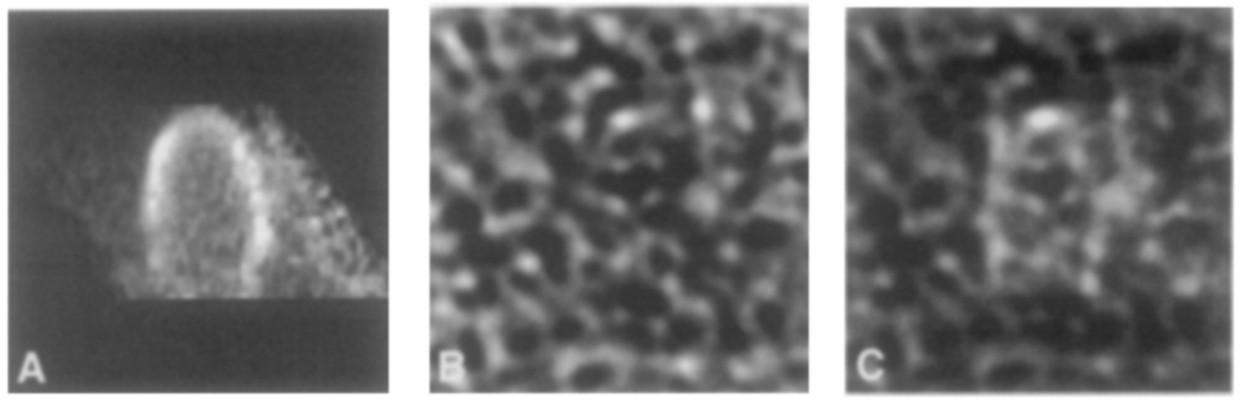

Figure 7. (A) FDG-PET, (B) SPECT noise, (C) PET-SPECT: typical example of smoothed FDG-PET image with rescaled SPECT noise added. Note that noise is so great that it is difficult to discern myocardium. Noise in PET-SPECT data was chosen to represent worst-case noise in actual $\mathrm{T}_{1}$ study.

sisted of patients of age range 40 to 65 years, mean $=58,50 \%$ men and women, who were studied because of severe heart pain. All patients were abnormally perfused, with either enlarged or hypoperfused myocardium.

For the validation experiments, we constructed a simulated Tl-201-SPECT image sequence from FDG-PET images. The FDG-PET images have good resolution and low noise, and the segmentation results could be taken as "truth" for comparison with the simulated Tl-201 data. To simulate the Tl-201 data, the PET data were first smoothed to $14 \mathrm{~mm}$ with a Gaussian filter to obtain the typical Tl-201 image resolution. Next, real tomographic SPECT noise was obtained by acquiring images of a uniform cylindrical phantom by the same SPECT scanner and at the same resolution as was used for the T1-201-SPECT image acquisitions. Multiple acquisitions were collected at multiple time points to give 50 different realizations of the same uniform noise. We chose the level of noise to produce a more noisy than average scenario (ie,

$$
\frac{\mu_{\text {myocardium }}}{\sigma_{\text {noise }}}=36 \%, \frac{\mu_{\text {tung }}}{\sigma_{\text {noise }}}=62 \%, \frac{\mu_{\text {bloodpool }}}{\sigma_{\text {noise }}}=49 \%,
$$

$\mu$ and $\sigma$ denote mean and standard deviation, respectively). The simulated Tl-201-SPECT sequences will be called PETSPECT, and an example is shown in Figure 7.

All 54 subjects (19 PET, 9 Tc-99m-MIBI-SPECT and 26 Tl201-SPECT) underwent resting planar gated blood pool imaging (20 to $25 \mathrm{mCi}$ Tc-99m-labled red blood cells) within approximately 1 week of SPECT perfusion or metabolism imaging. Imaging was performed in list mode, with 44 images per cycle, reverse framing, and $10 \%$ to $15 \%$ RR window for bad beat rejection. Six million counts were obtained over a $\sim 15 \mathrm{~cm}$ $\times 15 \mathrm{~cm}$ field of view, in a modified (10- to 15-degree caudal tilt) left anterior oblique projection. Ejection fraction was computed from the resultant 44-point LV volume curve and a background region (manually defined) as previously described. ${ }^{26}$ All EF computations were performed by individuals who had no knowledge of the gated SPECT results.

\section{Experiments}

In a first experiment, we proposed to assess accuracy of automatically determined LV contours with respect to manually obtained ones. We compared automatically obtained epicar- dial volumes versus volumes obtained through manual border tracing by two physicians for PET, Tl-201-SPECT and PETSPECT images. We also compared borders manually obtained by physician 1 with borders manually obtained by physician 2 and verified that the automatically obtained values lay within the range of uncertainty of the manual tracers. The experiment was repeated on a second day, several weeks after the first, with no possibility to refer to the previous experiment or to the drawing of the other physician. Hence, we were also able to study the intraobserver variability of the physicians. We further compared manually and automatically obtained volumes from the PET-SPECT images with the ones from the true underlying contours. We estimated the "true" values by averaging the values obtained from the underlying FDG-PET images by automatic and by manual border tracing by the 2 physicians. For all experiments, the physicians used a public domain image processing software (NIH Image V.1.60) to trace the boundaries using the mouse of a MacIntosh computer. Also, the same (manually determined) valves planes were used on both days and for both manually and computer-generated results to avoid introducing valve plane location variability. The epicardial contour was chosen because of its importance for EF computation in equation 4.

The next two experiments were designed to test the effectiveness of incorporating epicardial boundary information in $\mathrm{EF}$ computation. First, we tested the hypothesis that epicardial contours would exhibit less variability than endocardial contours. For this purpose we constructed 50 replicates of a PET-SPECT sequence with the same amount but with different samples of noise. We then compared the endocardial and epicardial volumes at one gating interval over all 50 replicates to determine the relative variability of the epicardial and endocardial borders.

Next, a second experiment was performed to evaluate the effectiveness of computing EF on the basis of the epicardial contour and myocardial volume (equation 4 ) versus a computation based only on the endocardial contour (equation 3). For this purpose we compared both methods of EF computation for the noise-free PET images and the noisy PET-SPECT images. It is also of interest to compare EF values for the PET images versus EF values obtained for the PET-SPECT images (both, of course, have the same underlying data and EF) for both equations 3 and 4 . The better the correlation, the more stable is the EF computation. Finally, we compared EFs obtained from rest 
Table 1. Comparison of absolute epicardial volumes obtained by manual and automatic segmentation

\begin{tabular}{llll}
\hline & \multicolumn{1}{c}{ PET } & \multicolumn{1}{c}{ T1-SPECT } & \multicolumn{1}{c}{ PET-SPECT } \\
\hline Day 1 & & & \\
p1 vs p2 & $y=19.2+0.86 x ; r=0.84$ & $y=-12.4+0.96 x ; r=0.78$ & $y=-12.8+1.0 x ; r=0.75$ \\
auto vs p1 & $y=3.24+0.94 x ; r=0.93$ & $y=30.8+0.75 x ; r=0.83$ & $y=25.2+0.81 \mathrm{x} ; \mathrm{r}=0.79$ \\
auto vs p2 & $y=-3.92+0.94 \mathrm{x} ; \mathrm{r}=0.90$ & $y=-0.76+0.87 \mathrm{x} ; \mathrm{r}=0.79$ & $y=-45.6+1.14 \mathrm{x} ; \mathrm{r}=0.80$ \\
Day 2 & & & \\
p1 vs p2 & - & $y=-0.84+0.89 \mathrm{x} ; \mathrm{r}=0.75$ & $y=24.6+0.78 \mathrm{x} ; \mathrm{r}=0.71$ \\
auto vs p1 & - & $y=40.8+0.76 \mathrm{x} ; \mathrm{r}=0.76$ & $y=18.8+0.93 \mathrm{x} ; \mathrm{r}=0.78$ \\
auto vs p2 & - & $y=5.95+0.91 \mathrm{x} ; \mathrm{r}=0.77$ & $y=6.64+0.95 \mathrm{x} ; \mathrm{r}=0.73$ \\
Days 1 and 2 & - & & \\
p1 vs p1 & - & $y=24.6+0.89 \mathrm{x} ; \mathrm{r}=0.80$ & $y=58.8+0.77 \mathrm{x} ; \mathrm{r}=0.67$ \\
p2 vs p2 & - & $y=14.7+0.98 \mathrm{x} ; \mathrm{r}=0.90$ & $y=31.3+0.84 \mathrm{x} ; \mathrm{r}=0.93$ \\
\hline
\end{tabular}

$p 1$, Physician $1 ; p 2$, physician 2; auto, automatically obtained contour.

Three test images (PET, 32 images, TI-SPECT, 40 images and PET-SPECT, 32 images) were analyzed on two different days separated by several days. Two independent and experienced physicians outlined the manually drawn contours. Comparison of the linear agreement between contours obtained by human beings and between contours obtained by a human beings versus automatically.

Table 2. Comparison of absolute volumes obtained from the "true" contours and manually and automatically determined contours

\section{PET-SPECT}

$\begin{array}{ll}\text { Day } 1 & \\ \text { True vs auto } & y=4.72+1.01 \mathrm{x} ; \mathrm{r}=0.95 ; \text { see }=5.2 \\ \text { True vs } \mathrm{p} 1 & y=19.2+0.87 \mathrm{x} ; \mathrm{r}=0.79 ; \text { see }=10.3 \\ \text { True vs } \mathrm{p} 2 & y=-48.2+1.20 \mathrm{x} ; \mathrm{r}=0.77 ; \text { see }=15.1 \\ \text { Day 2 } & \\ \text { True vs p1 } & y=25.0+0.93 \mathrm{x} ; \mathrm{r}=0.76 ; \text { see }=12.7 \\ \text { True vs p2 } & y=-5.28+0.98 \mathrm{x} ; \mathrm{r}=0.70 ; \text { see }=15.1\end{array}$

p1, Physician 1; p2, physician 2; auto, automatically obtained contour. Manual contours were drawn by two independent physicians on 2 different days.

GPB imaging with those obtained from the automatic algorithm (using equation 4) for FDG-PET, Tc-99m-SPECT, and T1201-SPECT images.

For all experiments, standard linear regression was performed, where $r$ denotes the correlation coefficient

$$
r=\frac{\operatorname{cov}(x, y)}{\sigma_{x} \cdot \sigma_{y}}
$$

with $\sigma_{x}$ and $\sigma_{y}$ denoting the standard deviation of $x$ and $y$. Also root mean square errors are given and denoted by see.

\section{RESULTS}

\section{Accuracy and Robustness Assessment}

Table I summarizes the results for assessment of the algorithm's accuracy. Experiment 1 (day 1) and experi- ment 2 (day 2) compare absolute epicardial volumes obtained by physician 1 versus physician 2 ; automatic computation versus physician 1 ; and automatic computation versus physician 2 for the PET, Tl-SPECT, and PETSPECT data. Row 3 presents the intraobserver agreement between day 1 and 2 for each human tracer. In all experiments, the results from the automatic segmentation algorithm lie within the range of uncertainty of the interobserver agreement. The results given in columns 2 and 3 of Table I (Tl-201-SPECT and PET-SPECT) indicate that the human uncertainty increases for noisy images, but the automatic algorithm still exhibits good relative accuracy with respect to manual results. The high correlation coefficients in column 1 of Table I (PET image) suggest that for these higher resolution, less noisy images, all of the contours are close to the true underlying contour. Hence, we considered the average of these three contours as the "true" contour for the comparisons described below.

Next we compared automatic and manual segmentation results on the PET-SPECT images to the true underlying contours as obtained from the far less noisy PET image. The corresponding results are given in Table II. The automatically obtained contours show a better linear agreement than the manually obtained ones, indicating better consistency. The correlation coefficients for the human tracers are quite low, presumably because of the very high noise level in the PET-SPECT images (Figure 7). The root mean square residual values (sees) for the automatic algorithm are much better than for the human tracers. A graphical representation of the true volumes versus automatically obtained ones is given in Figure 8.

One of the most valuable features of our method is its ability to handle very different types of images in an autonomous fashion. For all of the results presented in 


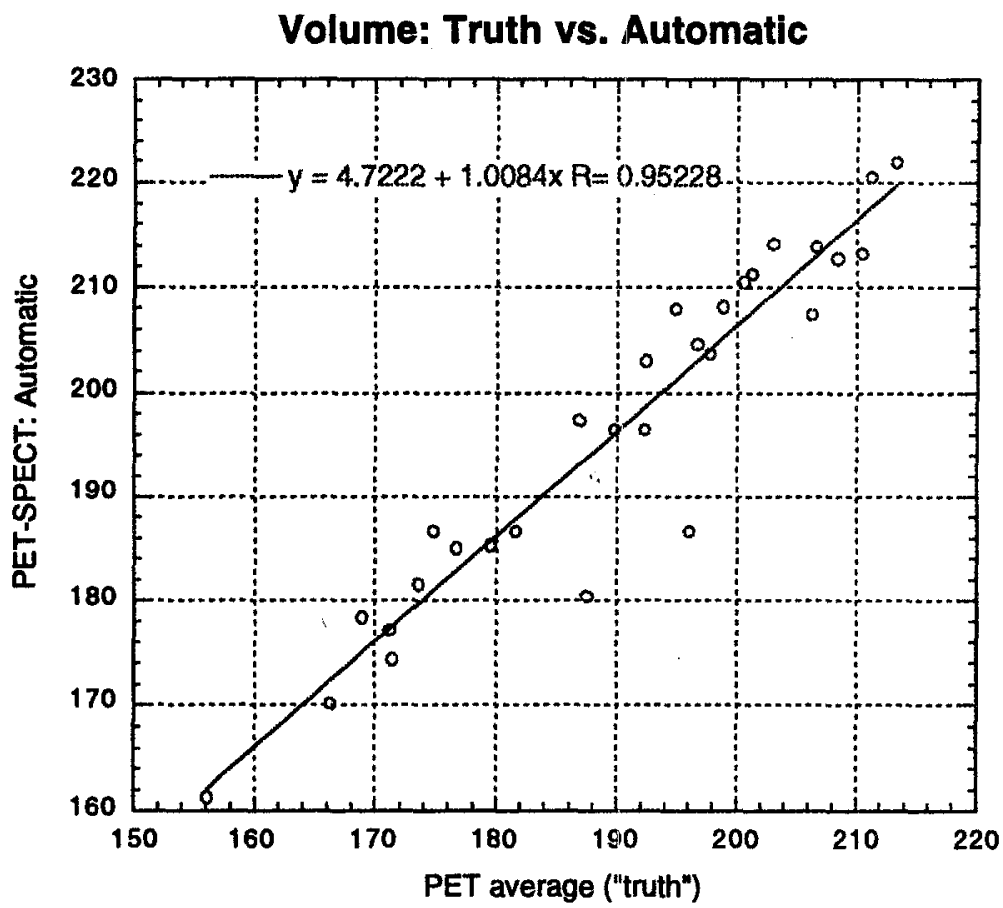

Figure 8. Comparison of "true" (average from FDG-PET) and automatically computed volumes.

Table 3. Comparison of variances in endocardial and epicardial volume computation with 50 replicates of the same PET-SPECT sequence with different noise realizations

\begin{tabular}{lcc}
\hline Estimate of & $\begin{array}{c}\text { Endocardial } \\
\text { volume }\end{array}$ & $\begin{array}{c}\text { Epicardial } \\
\text { volume }\end{array}$ \\
\hline $\begin{array}{l}\text { Number of } \\
\text { samples } \\
\begin{array}{l}\text { Mean value } \\
\text { Variance }\end{array}\end{array}$ & $\hat{\mu}=50$ & $n=50$ \\
\hline
\end{tabular}

this article, the exact same parameters were used in all steps of the segmentation and computation process, regardless of modality or image noise.

\section{Variability of the Method}

We correlated epicardial and endocardial volumes obtained from 50 replicates of a smoothed FDG-PET frame to which the same amount, but a different realization, of SPECT noise was added. We estimated the mean and variance for both endocardial and epicardial volumes, given in Table III. Variances were computed normalized to the same dynamic range in both data sets. Although these variances should not be used to estimate the true variance of either method (the same valve planes were used in all 50 replicates, no reslicing differences,
Table 4. Comparison of the formulas for $\mathrm{EF}$ on the basis of the endocardial contours

$\left[E F_{E N D O}=\frac{V_{\text {endo }}\left(t_{D}\right)-V_{\text {endo }}\left(t_{S}\right)}{V_{\text {endo }}\left(t_{D}\right)}\right]$ and on the epicardial contours $\left[E F_{E P I}=\frac{V_{\text {epl }}\left(t_{D}\right)-V_{\text {ep }}\left(t_{S}\right)}{V_{\text {epi }}\left(t_{D}\right)-m}\right]$

EF correlation

$\begin{array}{lll}\text { PET } & \text { EF }_{\text {ENDO VS EF }} & r=0.92, \text { see }=6.2 \\ \text { PET-SPECT } & \text { EF }_{\text {ENDO Vs EF }} & r=0.78, \text { see }=9.0 \\ \text { EF }_{\text {ENDO }} & \text { PET vS PET-SPECT } & r=0.76, \text { see }=8.7 \\ \text { EF }_{\text {EPI }} & \text { PET vS PET-SPECT } & r=0.92, \text { see }=5.3\end{array}$

Compared are EFs for the low noise PET image and for the noisy PET-SPECT image.

normalization to same dynamic range, etc), they can be used to examine differences in variance between the two methods. The $F$ distribution ${ }^{27}$ was used to establish significant difference between endocardial and epicardial variances. As shown in Table III the epicardial method had a significantly $(P<.005)$ smaller variance (over a factor of 2 smaller).

\section{Evaluation of EF on the Basis of Epicardial Contours}

Table IV compares EF computed from endocardial boundaries (with equation 3) versus EF computed from epicardial boundaries (with equation 4). The first row compares the results on the basis of the low noise origi- 


\section{PET: EF-endo vs. EF-epi}

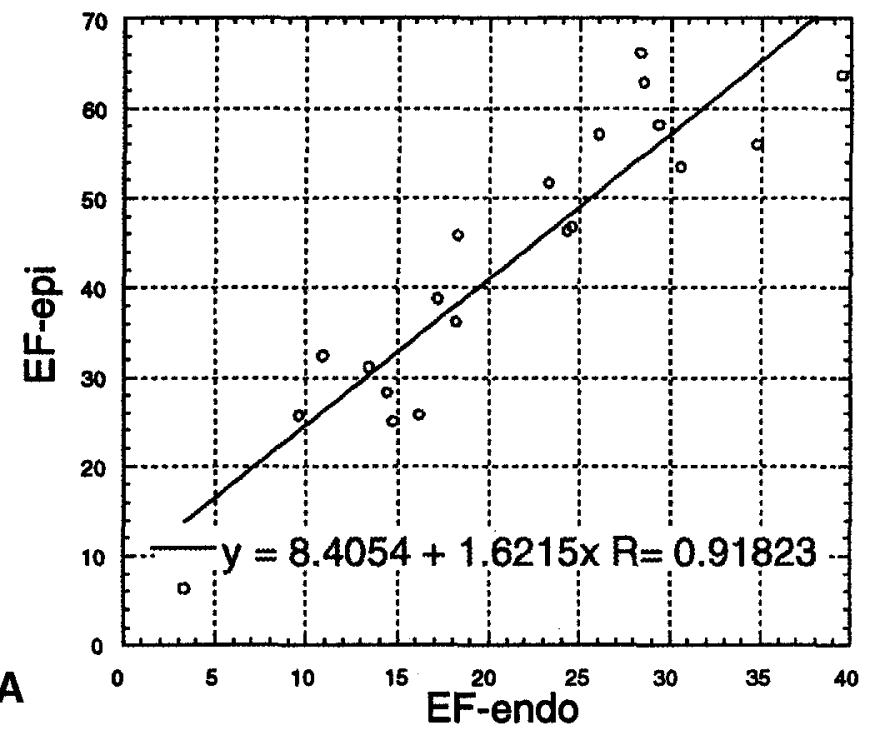

EF-endo: PET vs. PET-SPECT

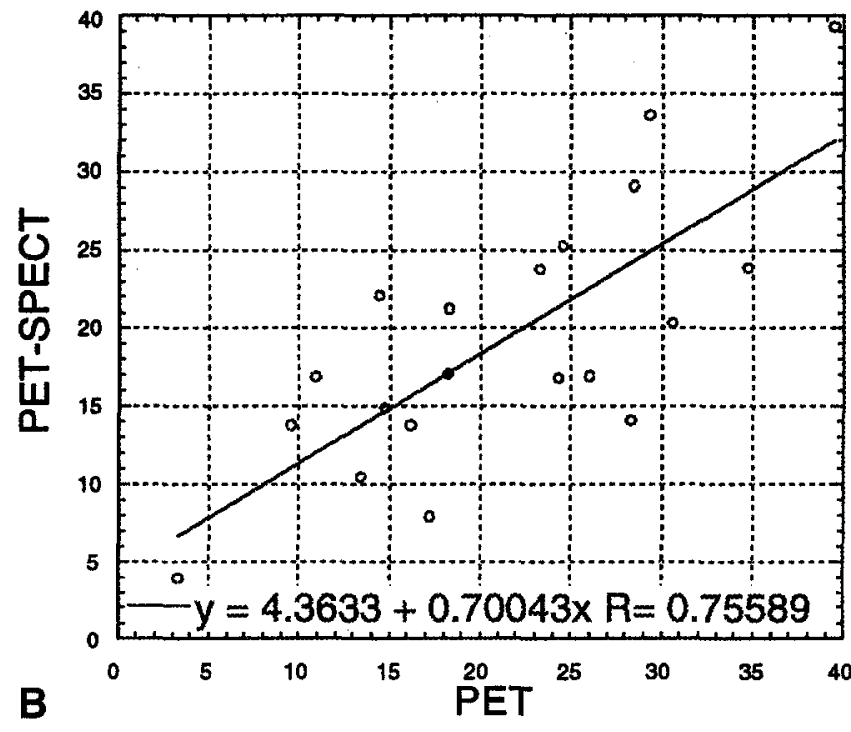

EF-epi: PET vs. PET-SPECT

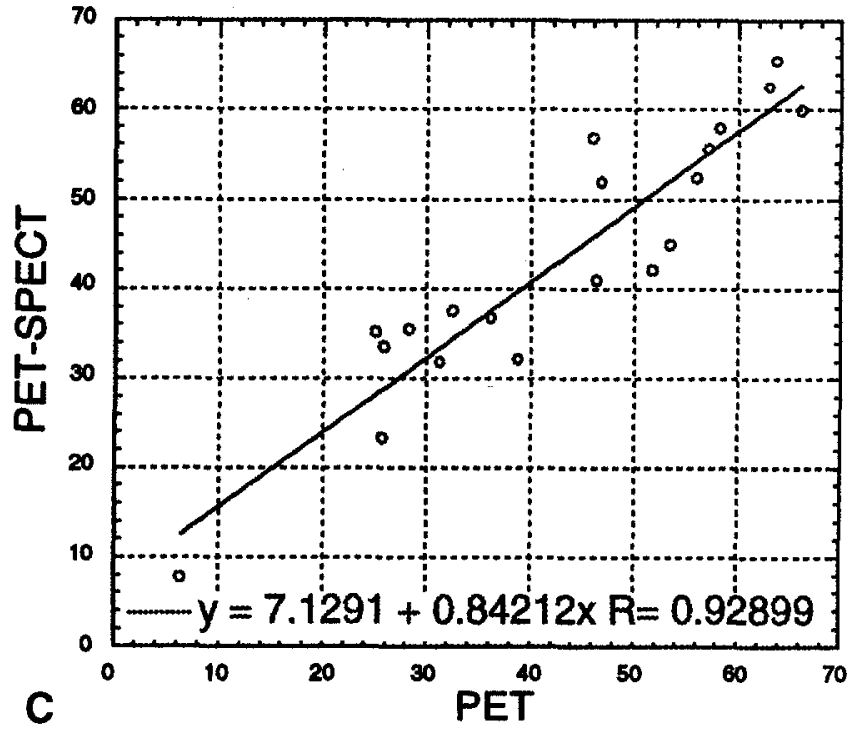

Figure 9. (A) PET image: EF-endo (equation 3) versus EF-epi (equation 4); (B) EF-endo: PET versus PET-SPECT; (C) EF-epi: PET versus PET-SPECT.

nal PET image, and the second row considers the noisy PET-SPECT image.

From row 1, one can see that the method of equation 4 agrees with the traditional endocardial-based method when the signal-to-noise ratio is high $(r=0.92)$. Hence, for less noisy images, both formulas 3 and 4 give essentially the same result (Figure 9,A, closer unity slope would be possible by parameter adjustment for matched filters). The second row of Table IV compares EF results on the basis of the noisy PET-SPECT images. As could be expected, the noisy data weaken the linear agreement between the two methods of measurement. Rows 3 and 4 give an indication with regard to which of the two measurements-endocardial or epicardial —is responsible for the drop in $r$ value observed between rows 1 and 2-that is, which of the techniques is likely to have higher variability. In rows 3 and 4 EFs obtained from the low-noise PET images and obtained from the noisy PET-SPECT images are compared by use of the same formula. Ideally, a unity correlation coefficient should be obtained. Clearly, compu- 

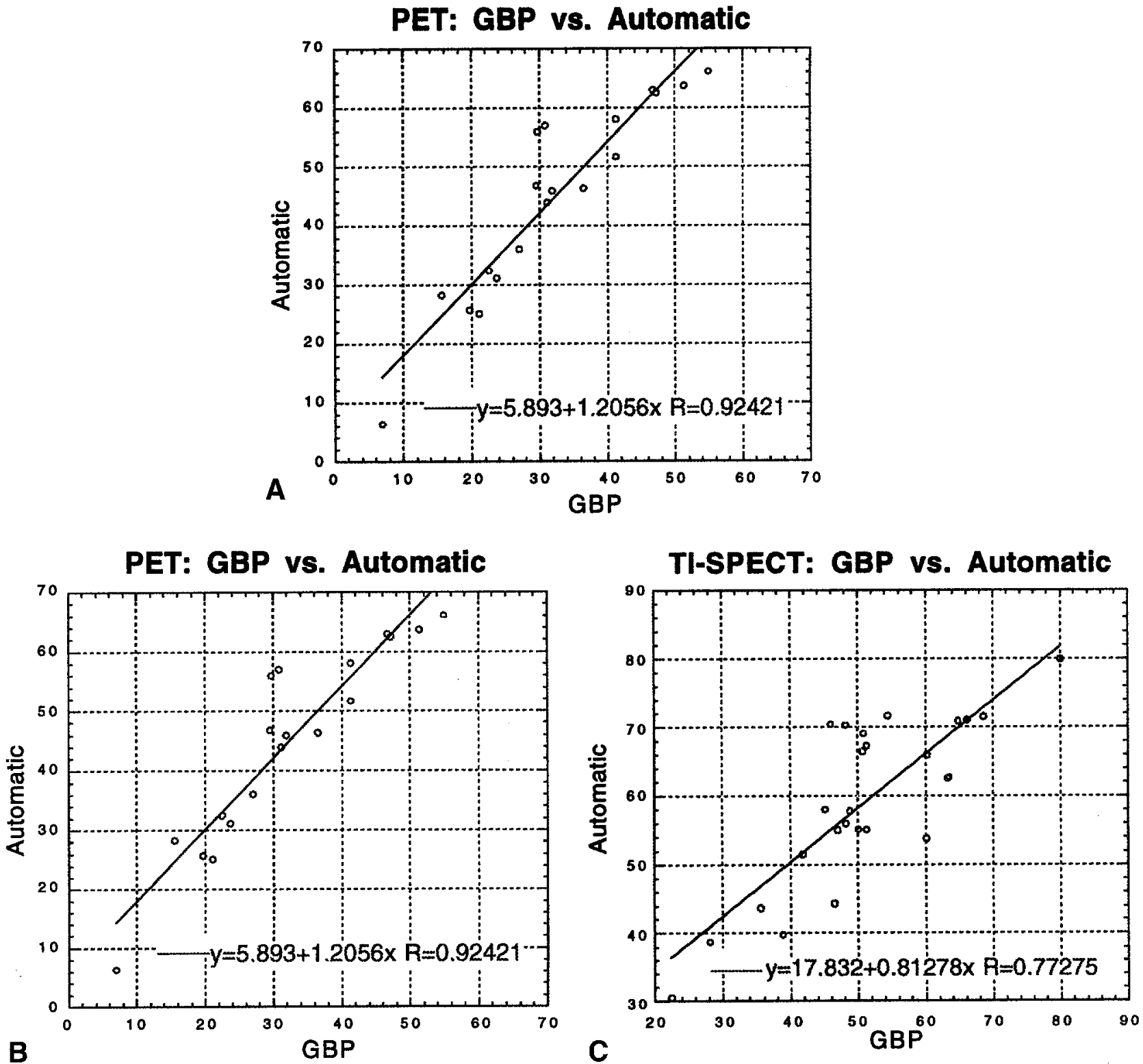

Figure 10. Graphical illustration of EF comparison GBP versus automatic algorithm.

tation of EF on the basis of the epicardial contour (equation 4 ) is more accurate for noisy images (ie, Figure $9, C$, is more highly correlated than Figure $9, B$ )-the change in $r$ value from 0.76 to 0.92 is significant at $P<.001$ ). For noiseless images, either formula 3 or 4 may be used.

\section{Comparison of Automatically Obtained EFs with GBP}

Figure 10 shows the relationship between the automatically computed EF measurements on gated FDGPET, Tc-SPECT and Tl-SPECT images and EFs obtained from planar GPB imaging. All the sets show a good linear agreement (FDG-PET: $y=5.89+1.21 x, r=$
0.92, see $=6.24, n=19 ;$ Tc-99m-SPECT: $y=-3.86+$ $1.06 x, r=0.88$, see $=7.78, n=9$; Tl-201-SPECT: $y=$ $17.8+0.81 x, r=0.77$; see $=7.44, n=26$ ). The difference in $r$ values between FDG-PET and Tl-201-SPECT were significant $(P<.001)$, whereas those between other pairs were not.

\section{DISCUSSION}

Only recently have algorithms emerged for a fully automatic exploitation of the intrinsic quantitative data contained in gated tomographic images. One of the first publications for CT images was by Dove et $\mathrm{al}^{28}$ using 
Fuzzy Hough transform combined with region growing and border detection techniques. Germano et al ${ }^{5}$ presented an automatic algorithm for EF computation for Tc99m-SPECT images. The images are thresholded and clustered to obtain a 3-dimensional binary mask of the left ventricular myocardium. Then an ellipsoid is fit to normal count profiles, which have been convolved with a feature detector, to obtain the heart boundaries. Recently the algorithms were extended to Tl-201-SPECT. ${ }^{6}$ Nichols et $\mathrm{al}^{4}$ have proposed another method to compute EF for Tc-99m-SPECT images on the basis of LV centering, detection of end-diastolic and-systolic frames and endocardial segmentation on the basis of count searching thresholding values. Threshold values were derived empirically from the results obtained by experienced physicians. Abstracts of two more examples of automatic EF computation for Tc-99m-SPECT can be found in references 13 and 29 where the underlying algorithms seem to be related to references 5 and 4 . We have described a semiautomatic algorithm that has a reduced dependency on empirically determined threshold values. A proper representation of the underlying image data and the use of stable contour tracking algorithms can meet such an objective. We used an elliptical coordinate transformation and track the contours with dynamic programming. The method does not require the myocardium to be of elliptical shape, however. The proposed method is suited for noisy environments, but it can also be used for better quality images. Our experiments show that the automatic algorithm produces results that lie well within the uncertainty of a manual border tracing (Table I). The linear agreement (ie, the $r$ value, not the closeness of the slope to unity) between the algorithm and each physician is better than the interphysician comparison $(P<.025)$. It suggests that each physician has a slightly different subjective perception of the correct boundary and that the algorithm tends to be an average of the manual results. For images with a high signal-to-noise ratio (PET), results for manual and automatic segmentation all agree very well, and the relative uncertainties are smaller in all cases.

The algorithm seems to provide a better representation with respect to the true contour than the manual analysis, as shown by Table II. The root mean square residual value (see) is significantly lower for the algorithm than for the human tracers. The relatively large see values in Table II reflect the extremely noisy nature of the simulated SPECT images. A true Tl-201-SPECT image sequence is likely to be of better quality than the simulated PET-SPECT sequence. Hence, we might expect at least similar or better correlation values and see values for the automatic algorithm and the "truth." On the basis of these reflections and the given results, we can also speculate that EF computation from T1-201-SPECT images indeed seems feasible.

To overcome the effect of gaps in the myocardial signal, integration of most probable offsets of endocardial points to mid-myocardial points is often used. ${ }^{5,30}$ The dynamic programming approach used here seems to deal successfully with this issue. In addition, we also deal with high noise in the signal by computation of EF on the basis of the epicardial contour, which constitutes a key result of our work. From our experimentation we conclude that EF based on the epicardial contours is more reliable for noisy images when using our algorithm. This EF method might also improve variability in other existing techniques. Note that results in Figure 9 demonstrate relative accuracy, and absolute accuracy is not studied. By adjusting the parameters used for the matched filters, one could obtain closer absolute values (ie, better unity slope). The correct calibration of the filters could be obtained by ascertaining optimum contour offsets via comparison of values from independent methods such as x-ray contrast angiography.

We have compared automatically computed EFs with EFs obtained from GBP images. Correlation factors for FDG-PET and Tc-99m-SPECT images, as well as see values, are consistent with those found by references 4 and 5 . Values for the Tl-SPECT images are acceptable and encourage bearing in mind the low signal-to-noise ratio of those images. Because the correlation value and see values reflect uncertainties of both the automatically obtained results and the values obtained from GBP, we can expect a better correlation with respect to the true underlying data. The algorithm copes well with perfusion defects, because of the implied smoothness constraint of the dynamic contour tracking. In the transformed coordinate system, a perfusion defect of length $l$ may only lead to a vertical drift of $d<l 2$ - $\nabla y$, where $\nabla y$ is the smoothness constraint in pixels.

The reader should be reminded that all comparisons of EFs made above were made with a manually selected valve plane. To test the ability of the method to produce robust myocardial contours (and therefore EFs), the same valve plane was used when comparing the endocardial method of equation 3 with the epicardial method of equation 4 . By so doing, we were able to compare the relative variabilities of the two EF methods isolated from other sources of variability. It should be noted that to make use of the proposed method in clinical practice, one would presumably use any of a number of commercially used valve plane selection methods. ${ }^{4,5}$ It should also be noted that the ability of this proposed method to measure absolute volumes has not been tested and would, as with all previously described methods, depend not only on the accuracy of the contour selection, but also on the accuracy of valve plane selection.

\section{CONCLUSIONS}

We have presented new image processing methods for reliable contour segmentation, as well as an alternative method for EF computation, from gated PET, Tc-SPECT, 
and Tl-SPECT images. The segmentation uses an elliptical coordinate system to facilitate contour enhancement and contour detection but does not require an elliptically shaped heart. The actual segmentation is performed by dynamic programming, which results in very efficient computation and produces smooth and connected contours. The segmentation is visually accurate in practically all of the images analyzed and appears to produce reliable values of $\mathrm{EF}$ even for noisy images. An important feature of the algorithm is that there are few parameters, and these do not require modification for different image modalities or different image qualities (eg, noise or resolution). We have proposed an approach for EF computation on the basis of both the epicardial and endocardial contour. It relies on the physiological constraint of constant myocardial volume. Our experiments show that this EF method produces an excellent correlation with the traditional method of EF computation for images with high signalto-noise ratios and reduced variance for images with low signal-to-noise ratio. This EF method could easily be adapted for use in other existing techniques.

\section{References}

1. DePuey E. Evaluation of cardiac function with radionuclides. In: Gottschalk HP, ed. Diagnostic nuclear medicine. Baltimore: Williams \& Wilkins, 1988:355-98.

2. Barat J, Brendel A, Colle J, et al. Quantitative analysis of left-ventricular function using gated single photon emission tomography. J Nucl Med 1984;25:1167-74.

3. Caputo G, Graham M, Brust K, Kennedy J, Nelp W. Measurement of left ventricular volume using SPECT. Am J Cardiol 1985;56:781-6.

4. Nichols K, Puey EGD, Rozanski A. Automation of gated tomographic left ventricular ejection fraction. J Nucl Cardiol 1996;3:475-82.

5. Germano G, Kiat H, Kavanagh P, Moriel M, Mazzanti M, Su H, Train $\mathrm{KV}$, Berman D. Automatic quantification of ejection fraction from gated myocardial perfusion SPECT. J Nucl Med 1995;36:2138-47.

6. Germano G, Erel J, Kiat H, Kavanagh P, Berman D. Quantitative LVEF and qualitative regional function from gated thallium-201 perfusion SPECT. J Nucl Med 1997;38:749-54.

7. Yang KA, Chen HD. A semi-automated method for edge detection in the evaluation of left ventricular dunction using ECG-gated single-photon emission tomography. Eur J Nucl Med 1994;21:1206-11.

8. Bartlett ML, Buvat I, Vaquero JJ, Mok D, Dilsizian V, Bacharach SL. Measurement of myocardial wall thickening from PET/SPECT images: comparison of two methods. J Comput Assist Tomogr 1996;20:473-81.

9. Mok DY, Bartlett ML, Bacharach SL, Voipio LM, Carson RE, et al. Can partial volume effects be used to measure myocardial thickness and thickening? Comput Cardiol 1992;195-8.

10. Porenta G, Kuhle W, Sinha S, Krivokapich J, Czernin J, Gambhir SS, et al. Parameter estimation of cardiac geometry by ECG-Gated PET imaging: validation using magnetic resonance imaging and echocardiography. J Nucl Med 1995;36:1123-9.

11. Galt JR, Garcia EV, Robbins WL. Effects of myocardial wall thickness on SPECT quantification. IEEE Trans Med Imaging 1990;9:144-50.
12. Yamashita K, Tamaki N, Yonekura Y, Ohtani H, Magata Y, Nohara R, et al. Regional wall thickening of left ventricle evaluated by gated positron emission tomography in relation to myocardial perfusion and glucose metabolism. J Nucl Med 1991;32:679-85.

13. Vansant JP, Faber TL, Folks RD, Nichols K, DePuey G, Garda E. Validation of an automated methodology for determining left ventricular ejection fraction from gated SPECT. J Am Coll Cardiol 1997;29(suppl A):263A.

14. Duda RO, Hart PE. Use of the Hough transformation to detect lines and curves. Commun ACM 1972;29:11-5.

15. Brigger $P$, Unser $M$. General discrete image pyramids. SPIE 1997;3169:212-23.

16. Hoffman EI, Huang SC, Phelps ME. Quantitation in positron emission computed tomography: 1 - effect of object size. I Comput Assist Tomogr 1979;3:299-308.

17. Jain AK. Fundamentals of digital image processing. Englewood Cliffs, NJ: Prentice-Hall, Inc., 1989.

18. Unser M, Pelle G, Brun P, Eden M. Automated extraction of serial myocardial borders from M-mode echocardiograms. IEEE Trans Med Imaging 1989; MI-8:96-103,

19. Marcassa C, Marzullo P, Parodi O, Sambuceti G, L'Abbate A. A new method for noninvasive quantitation of segmental myocardial wall thickening using technetium-99m 2-methoxy-isobutyl-isonitrile scintigraphy-results in normal subjects. J Nucl Med 1990;31:173-7.

20. Mochizuki T, Murase K, Fuji Y. Assessment of systolic thickening with thallium 201 ECG-gated single-photon emission computed tomography: a parameter for local left ventricular function. J Nucl Med 1991;32:1496500.

21. Cooke CD, Garcia EV, Cullom SJ, Faber TL, Pettigrew RI. Determining the accuracy of calculating systolic wall thickening using a fast Fourier transform approximation: a simulation study based on canine and patient data. J Nucl Med 1994;35:1185-92.

22. Buvat I, Bartlett ML, Kitsiou AN, Dilsizian V, Bacharach SL. A "hybrid" method for measuring myocardial wall thickening from gated PET/SPECT images. J Nucl Med 1997;38:324-9.

23. Bellman R. Dynamic programming. Princeton: Princeton University Press, 1957.

24. Ferrand S, Carson I, Brigger P, Bacharach S, Davis C, Dilsizian V. Effect of left ventricular valve plane selection on computation of left ventricular ejection fraction from gated thallium SPECT. Society of Nuclear Medicine, 45th Annual Meeting, 1998. Accepted for publication.

25. Bacharach SL, Green MV, Vitale D, White G, Douglas MA, Bonow RO, et al. Optimum Fourier filtering of cardiac data: a minimum-error method-concise communication. J Nucl Med 1983;24:1176-84.

26. Borer J, Bacharach S, Green M, et al. Real-time radionuclide cineangiography in the noninvasive evaluation of global and regional left ventricular function at rest and during exercise in patients with coronaryartery disease. N Engl J Med 1977;296:839-44.

27. Barlow RJ. Statistics: a guide to the use of statistical methods in the physical sciences. West Sussex, United Kingdom: John Wiley \& Sons; 1989.

28. Dove EL, Philip K, Gotteiner NL, Vonesh JL, Reed JARJE, Stanford W, et al. A method for automatic edge detection and volume computation of the left ventricle form ultrafast computed tomographic images. Invest Radiol 1994;29:945-54.

29. Bateman TM, Case JA, Saunders MJ, O'Keefe JH, Sherwani MEWK, Barnhart CS. Gated SPECT LVEF measurements using a dual-detector camera and a weight-adjusted dosage of thallium-201. J Am Coll Cardiol 1997;29(suppl A):263A.

30. Everaert H, Bossuyt A, Franken P. Left ventricular ejection fraction and volumes from gated single photon emission tomographic myocardial perfusion images: comparison between two algorithms working in three-dimensional space. J Nucl Cardiol 1997;4:472-6. 